\title{
The effects of zinc amino acid complex supplementation on the porcine host response to Lawsonia intracellularis infection
}

\author{
Fernando L. Leite ', Erika Vasquez², Fabio A. Vannucci², Connie J. Gebhart ${ }^{1}$, Aaron Rendahl', Jerry Torrison², \\ Adam Mueller ${ }^{3}$, Nathan L. Winkelman ${ }^{3}$, Zachary J. Rambo ${ }^{4}$ and Richard E. Isaacson ${ }^{1 *}$
}

\begin{abstract}
Lawsonia intracellularis is among the most important enteric pathogens of swine and antibiotic alternatives are needed to help mitigate the negative effects of infection. Zinc is an essential trace mineral known to be crucial for maintaining intestinal barrier function and proper immune response. In this study, we investigated the porcine host response to $L$. intracellularis infection when supplemented with a zinc-amino acid complex, a form of zinc that can lead to greater bioavailability when compared to traditional inorganic forms of zinc. Our results show that a zincamino acid complex supplementation with a final concentration of $125 \mathrm{ppm}$ of zinc in feed significantly $(p<0.05)$ decreased the number of animals with lesions and severity of lesions caused by L. intracellularis. Animals supplemented with the zinc-amino acid complex also exhibited a significantly $(p<0.05)$ earlier onset of seroconversion as well as an increased number of T cells in infected and non-infected intestinal tissue. This study demonstrated that this zinc-amino acid complex aids the host in responding to L. intracellularis infection and may be a new approach to help minimize negative effects of disease.
\end{abstract}

\section{Introduction}

Zinc is an essential mineral for animals and is a critical component of many structural proteins, enzymatic processes, and transcription factors [1]. Additionally, it is known that zinc has an important role that can influence both innate and adaptive immune responses [2]. In the intestine, zinc is essential for maintaining normal barrier function and important in regeneration of injured tissue $[3,4]$. Zinc can promote intestinal epithelial wound healing by enhancement of epithelial cell restitution, the initial step of epithelial wound healing [4]. In pigs, it has been demonstrated that a zinc-amino acid complex (Availa Zn) can improve trans-epithelial resistance and help maintain epithelial cell morphology in the presence of a stressor such as heat stress [5].

\footnotetext{
*Correspondence: isaac015@umn.edu

${ }^{1}$ Department of Veterinary and Biomedical Sciences, College

of Veterinary Medicine, University of Minnesota, St. Paul, MN, USA

Full list of author information is available at the end of the article
}

Lawsonia intracellularis is an obligate intracellular pathogen that replicates inside of intestinal, primarily ileal, enterocytes and causes the disease porcine proliferative enteropathy (PPE) [6]. This disease has two major clinical forms: porcine intestinal adenomatosis (PIA) and proliferative hemorrhagic enteropathy (PHE). PIA is the most common form of this disease and, though often characterized as mild diarrhea, is also often characterized as a subclinical manifestation leading to decreased production performance [7]. L. intracellularis infection leads to lesions characterized by hyperplasia of enterocytes in intestinal crypts and compromises intestinal function, leading to decreased weight gain and feed conversion efficiency $[7,8]$.

Considering the importance of zinc in intestinal health, it is important that it be properly supplemented. Zinc complexed with amino acids can lead to greater bioavailability as compared to inorganic forms $[9,10]$. High concentrations (2000-3000 ppm) of inorganic zinc oxide have been used in swine production for treatment and prevention of diarrhea in weaning piglets but have also 
been associated with negative impacts such as promoting antimicrobial resistance $[11,12]$. The objective of this study was to investigate the ability of zinc amino acid complex to meet the physiologic needs of the animal and its impact on the response to L. intracellularis infection. In this study, we compared zinc provided in an amino acid complex formulation (ZnAA) to supplementation with inorganic zinc sulfate alone. The dose used in this study was $125 \mathrm{ppm}$, which was selected because it is close to the daily requirement of swine (estimated to be around $100 \mathrm{ppm})$ [13, 14]. We additionally investigated the impact of providing $\mathrm{ZnAA}$ in water as compared to feed.

\section{Materials and methods}

\section{Study design and animal diets}

To study the impact of zinc amino acid complex provided in feed (ZnAA) (Availa Zn, Zinpro Corporation) and water (ZnAALQ) (Availa Zn LQ, Zinpro Corporation) on pigs challenged with $L$. intracellularis, a total of 4 treatment groups were used in a randomized block design. A total of 72 4-week-old pigs were randomized by weight and gender and assigned to one of four treatment groups. The pigs were of a Landrace cross to a Yorkshire female with a large white sire (Topigs-Norsvin). All pigs were negative for L. intracellularis infection prior to the start of the study. There were 18 pigs per treatment divided among 6 pens with three animals per pen (Table 1 ). The pens were equally distributed in two barns (barn A and barn B). All pigs were fed their respective diets ad libitum 21 days prior to challenge with $L$. intracellularis. Diets in all treatments were formulated to meet or exceed phase requirements [14] and were isocaloric, isonitrogenous and iso for calcium, available phosphorus levels differed only in the source of $\mathrm{Zn}$ and $\mathrm{Mn}$. The negative and positive control groups received supplementation with $125 \mathrm{ppm}$ zinc sulfate, while one of the treatment groups received the same diet but with ZnAALQ added on top of the control diet via water (diluted to provide $1.09 \mathrm{mg}$ of ZnAALQ per $29.6 \mathrm{~mL}$ of water) while the other treatment group received $\mathrm{ZnAA}$ in feed at $50 \mathrm{ppm}$ and another $75 \mathrm{ppm}$ of zinc sulfate (a total of $125 \mathrm{ppm}$ ). The analyzed $\mathrm{Zn}$ concentration for the diet containing $\mathrm{ZnAA}$ was $200 \mathrm{mg} \mathrm{Zn/kg}$ and the analyzed concentration of the diet containing only $\mathrm{ZnSO}_{4}$ was $189.7 \mathrm{mg} \mathrm{Zn} / \mathrm{kg}$. All groups except the negative control group were challenged orally with a mucosal homogenate from a finishing pig with acute PHE, following a protocol previously described [15]. Briefly, to obtain the homogenate, mucosa was scraped from the small intestine of a pig affected with PPE lesions, and suspended at a 1:1 ratio in sucrose-phosphate-glutamate (SPG) buffer, $\mathrm{pH} 7.0$, which contained sucrose $(0.218 \mathrm{M})$, monobasic potassium phosphate $(0.0038 \mathrm{M})$, dipotassium phosphate $(0.0072 \mathrm{M})$, and L-glutamic acid $(0.0047 \mathrm{M})$ [15]. The pig that was used for the challenge homogenate was screened for other known swine pathogens and none were found. Animals were challenged with a dose of about $8.0 \times 10^{7} \mathrm{~L}$. intracellularis organisms by oral gavage at 7 weeks of age. Challenge dose was estimated by real time PCR. Groups of 6 pigs were randomly selected from each group of pigs per treatment and were euthanized at 14, 21 and 28 days post-challenge by electrocution.

\section{Animal production measurements}

To investigate the impact of zinc supplementation with ZnAA and ZnAALQ on the production performance of animals when infected with $L$. intracellularis, the average daily weight gain (ADG), average daily feed intake (ADFI), gain to feed ratio (G:F), and water disappearance were calculated. Animals were weighed weekly and recording of feed and water provided was recorded daily. Water disappearance was calculated by weighing water volumes every time water was provided to estimate the amount of water that had disappeared within a timeframe. ADG, ADFI, and water disappearance were calculated by measuring the difference of animal weight and feed and water consumed between each week dividing the difference by 7 to obtain daily averages for each week of the trial. For ADFI and water disappearance, the value was then divided by the number of animals per pen to obtain a per animal average. This calculation still considers the pen as the experimental unit. Gain to feed ratio was obtained by dividing ADG by ADFI values.

\section{Clinical observations}

Clinical signs were recorded throughout the experimental period and animals were evaluated for demeanor

Table 1 Animal treatments

\begin{tabular}{llllll}
\hline Treatment & Pens/treatment & Pigs/pen & Pigs/treatment & Zn level (ppm) and source & Challenge \\
\hline Positive control & 6 & 3 & 18 & $125, \mathrm{SO}_{4}$ & Yes \\
ZnAA in feed & 6 & 3 & 18 & $75 \mathrm{SO}_{4}+50$ Availa ZN & Yes \\
ZnAALQ in water & 6 & 3 & 18 & $125 \mathrm{SO}_{4}+$ Availa ZN LQ & Yes \\
Negative control & 6 & 3 & 18 & $125, \mathrm{SO}_{4}$ & No \\
\hline
\end{tabular}


score, abdominal score and fecal score. All scores used for evaluation varied from zero to three with increasing severity of clinical signs. Demeanor scores ranged from zero to three with 0 representing normal animal behavior and 3 being severely depressed/partially recumbent. Abdominal scores of zero represented normal and scores of three represented severely gaunt animals. A fecal score of zero represented no diarrhea, a score of one represented semi-solid/not formed feces, a score of two represented watery stool with less than $50 \%$ of water and a score of three represented profuse projectile diarrhea.

\section{Gross and microscopic pathology evaluation}

At 14, 21 and 28 days post-infection (dpi) groups of animals $(n=6)$ were randomly selected from each treatment group and were euthanized. Post-mortem examination of the ileum, jejunum, cecum and colon of all animals was carried out to identify the presence of gross pathologic change including those characteristic of PPE. Each lesion was scored as either mild (score of 1), moderate (score of 2) or severe (score of 3). For histopathology and evaluation of microscopic lesions characteristic of PPE, a section of the ileum proximal to the ileocecal valve was collected from all animals. Tissues were immediately fixed in formalin and then embedded in paraffin, and cut. The terminal ileum was selected for sampling since it is the most consistent site of $L$. intracellularis infection and lesions [16]. Histological evaluation was performed after staining with hematoxylin and eosin (HE) and immunohistochemistry (IHC) was performed using rabbit anti- $L$. intracellularis specific polyclonal antibody to measure the extent of proliferative lesions and to quantify the amount of $L$. intracellularis present in the tissues, respectively. The presence of $L$. intracellularis-specific antigen was measured blindly with a five-grade IHC scoring scale (grade 0 equal to absence of antigen in tissue; and grade 4 equal to all enteric crypts containing antigen) as previously described [17]. The extent of lesions was measured on a 3 point grading scale representing the distribution of crypt dysplasia with 1 assigned for focal lesions, 2 for multifocal lesions, and 3 for diffuse lesion distribution.

\section{Evaluation of $B$ cell distribution and $T$ cell quantification}

Since zinc is known to affect the immune system, we quantified $\mathrm{T}$ cell and $\mathrm{B}$ cell populations to determine if there were potential changes in immune responses at the site of infection based on cell population changes. For this, antibodies against CD3 ( $\mathrm{T}$ cell) and CD79a (B cell) were used. After blocking nonspecific binding sites with $10 \%$ normal goat serum for $15 \mathrm{~min}$, slides were incubated with primary antibodies for 30-45 min at room temperature. Slides were then rinsed with trisbuffered saline with $0.05 \%$ Tween 20 and incubated with horseradish peroxidase conjugated secondary antibodies to allow visualization of cells in tissue. The slides were then observed at $100 \times$ and $400 \times$ magnification using a light microscope. The distributions of B cells were scored on a 1-4 scale as follows: a focal distribution was given a score of 1 , a multifocal distribution given a score of 2 , a multifocal-to-diffuse distribution given a score of 3 and a diffuse distribution given a score of 4 . To quantify $\mathrm{T}$ cells, 60 crypts per animal were selected, 30 infected with $L$. intracellularis and 30 non-infected. The total number of $\mathrm{T}$ cells present in each crypt was measured and averaged per animal.

\section{Antibody response and fecal shedding}

To investigate the antibody response of the pigs to $L$. intracellularis, serum samples were collected by venipuncture of the cranial vena cava at 14, 21 and $28 \mathrm{dpi}$. Serum samples were serially diluted from $1: 30$ to $1: 1920$ in phosphate-buffered saline containing $2.5 \%$ fetal bovine serum, $1.0 \%$ rabbit serum and $0.08 \%$ tween 80 and screened for antibodies against $L$. intracellularis using the immunoperoxidase monolayer assay (IPMA) [18]. To measure shedding of L. intracellularis, fecal samples were collected at the same time points and DNA was extracted. Real time PCR was then used to estimate the quantity of $L$. intracellularis using standardized methods at the University of Minnesota Veterinary Diagnostic Laboratory.

\section{Statistical testing}

Statistical tests were performed using $\mathrm{R}$ [version 3.3.3 (2017-03-06)] using a mixed model and differences in lsmeans evaluated for statistical significance. The pen was considered the experimental unit for all assessments. $p$ values below 0.05 were considered significant. Trends were described as statistical results with $p$ values below 0.1 . To compare samples from positive and negative animals for seroconversion, gross lesions, IHC and HE lesions, the $\mathrm{N}-1$ Chi squared test was used.

\section{Results}

\section{Clinical measurements of disease}

There was only one animal with a fecal score above zero following challenge with $L$. intracellularis and that animal had a score of one indicating that it had a loose stools. This occurred at $19 \mathrm{dpi}$ and the animal was in the positive control group. All animals in the negative control group remained negative for $L$. intracellularis infection. There were no cases of diarrhea or clinical signs characteristic of PPE throughout the experimental period, thus any disease induced was subclinical. 


\section{Production measurements}

Average daily gain varied from an overall average of $0.56 \mathrm{~kg}$ per day at day 14 of the trial to $0.92 \mathrm{~kg}$ per day at the last time point $(28 \mathrm{dpi})$ regardless of treatment (Table 2). There were no statistical differences between treatments when comparing at a single time point.

For average daily feed intake, there was a barn effect as barn B had a higher overall feed intake average of $1.35 \mathrm{~kg}$ compared to $1.23 \mathrm{~kg}$ in barn A $(p<0.05)$. There were no trends nor statistical differences observed in ADFI when comparing treatments (Additional file 1). Based on average daily feed intake and analyzed $\mathrm{Zn}$ concentration, on average animals consumed $252.85 \mathrm{mg}$ of total zinc daily.

There was a trend for the group treated with $\mathrm{ZnAA}$ in feed to have a lower gain to feed ratio compared to the negative control group at $28 \mathrm{dpi}$ (Additional file 2). These ratios were of 0.438 and 0.502 , respectively $(p<0.1)$. Similar to ADG and ADFI, there were no statistical differences between treatments. Again, there was a difference between both barns, which was of 0.051 averaged over all time points $(p<0.05)$.

Water disappearance ranged from $1.46 \mathrm{~L}$ on day 7 of the study $(-14 \mathrm{dpi})$ to $3.81 \mathrm{~L}$ on average per animal at the last time point of the study (28 dpi) (Additional file 3). There were no barn effects or statistical differences between treatments. Statistical significance was only found comparing different time points overtime. Based on water disappearance, animals provided zinc in water consumed an additional $56.14 \mathrm{mg}$ of ZnAALQ on day 7 and $139.53 \mathrm{mg}$ on day $28 \mathrm{dpi}$ via water disappearance.

\section{Gross lesions}

No pigs had gross lesion scores consistent with PPE at $14 \mathrm{dpi}$. On both 21 and $28 \mathrm{dpi}$, pigs in both the ZnAA and ZnAALQ treatment groups had fewer animals with gross lesions compared to pigs in the positive control group (Figure 1A). At $21 \mathrm{dpi}, 33 \%$ of animals that received either ZnAA treatment had gross lesions ( 2 of 6 animals) while $66 \%$ of animals in the positive control group had gross lesions ( 4 of 6 animals). At $28 \mathrm{dpi}$, the
ZnAA group had significantly less animals with lesions $(p<0.05)$ compared to the positive control group with $33 \%$ (2 of 6 ) and $100 \%$ (6 of 6) of animals with lesions, respectively. At this time point, the ZnAALQ treatment led to a trend $(p<0.1)$ in reduction in the number of animals with gross lesions compared to the positive control group having $50 \%$ of animals with lesions (3 of 6). Both ZnAA and ZnAALQ treatments led to a significant $(p<0.05)$ reduction in lesion severity at $28 \mathrm{dpi}$ with the average lesion score being 1.33 in the positive control group compared to 0.33 in the $\mathrm{ZnAA}$ and 0.5 in the ZnAALQ group (Figure 1B).

\section{Immunohistochemistry and microscopic lesions}

To estimate the amount of L. intracellularis present in the intestinal tissue, IHC was performed. All treatment groups except the negative control group had $L$. intracellularis antigen present in tissue at 14, 21 and $28 \mathrm{dpi}$. The IHC score for both the ZnAA and the positive control groups peaked at $21 \mathrm{dpi}$ while the ZnAALQ group had similar IHC scores at 21 and 28 dpi. At $28 \mathrm{dpi}$, the group treated with ZnAA had the lowest IHC score with an average score of 0.67 compared to the positive control and ZnAALQ groups which had scores of 1.6 and 1.58 , respectively (Figure $2 \mathrm{~A}$ ).

In addition to determining the extent of infection by measuring bacterial presence, the extent of microscopic lesions was determined using paraffin embedded samples that were stained with HE. Trends observed in microscopic lesion severity were similar to those for IHC score (Figure 2C). The number of animals with microscopic lesions after receiving $\mathrm{ZnAA}$ in feed was significantly reduced $(p<0.05)$ compared to the number of animals in the positive control and ZnAALQ treatment groups with lesions. At $28 \mathrm{dpi}$, only $17 \%$ (1 of 6) of animals in the ZnAA group had lesions compared to $83 \%$ (5 of 6 ) of pigs in the positive control and ZnAALQ groups, respectively (Figure $2 \mathrm{~B}$ ).

Table 2 Average daily gain

\begin{tabular}{lllllll}
\hline Treatment & $\mathbf{- 7} \mathbf{~ d p i}$ & $\mathbf{0 ~ d p i}$ & $\mathbf{7 ~ d p i}$ & $\mathbf{1 4} \mathbf{d p i}$ & $\mathbf{2 1} \mathbf{d p i}$ & $\mathbf{2 8} \mathbf{d p i}$ \\
\hline Positive control & 0.58 & 0.69 & 0.83 & 0.74 & 0.87 & 0.91 \\
Negative control & 0.54 & 0.66 & 0.80 & 0.84 & 0.92 & 0.78 \\
ZnAA LQ & 0.58 & 0.77 & 0.86 & 0.75 & 0.90 & 0.88 \\
ZnAA & 0.55 & 0.73 & 0.84 & 0.04 & 0.005 & 0.80 \\
Standard error & 0.04 & 0.04 & 0.04 & 0.06 \\
\hline
\end{tabular}

Average daily gain was calculated in $\mathrm{kg}$ by measuring the difference of animal weight between each week of the experiment dividing the difference by 7 to obtain daily averages for each week of the experiment. Reported are the Ismeans, and the standard error of each time point. No significant differences were found between treatments but only between time points $(p<0.05)$. 

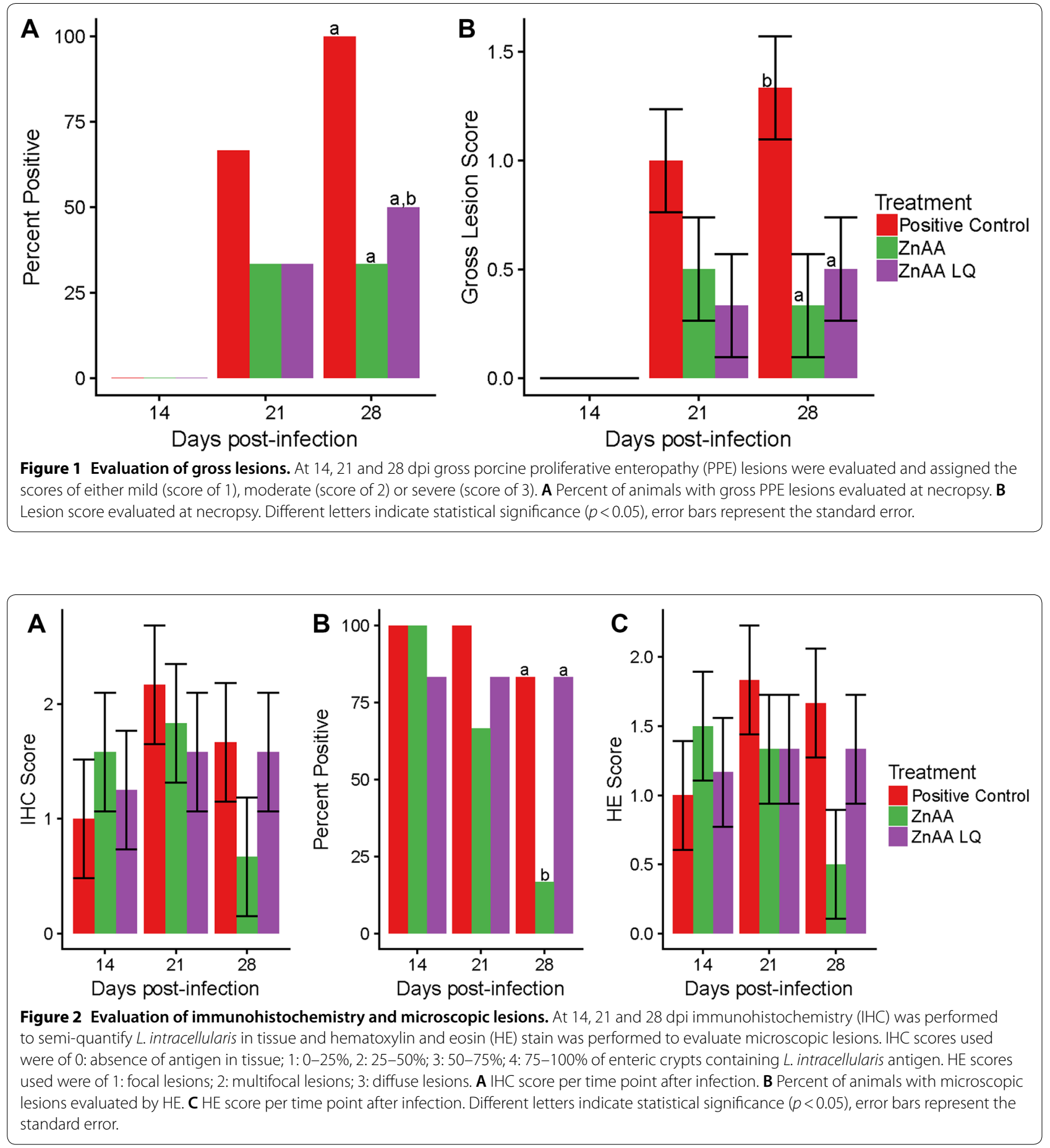

\section{Quantification of $L$. intracellularis shedding and serum antibody response}

Since $L$. intracellularis antigen in tissues was reduced in pigs given with $\mathrm{ZnAA}$, we next investigated if $\mathrm{ZnAA}$ supplementation reduced shedding of $L$. intracellularis in feces. Therefore, real time PCR to detect $L$. intracellularis in feces was performed on samples collected at 14, 21 and 28 dpi. No differences in fecal shedding were observed between treatment groups (Additional file 4). A Ct value below 35 was considered a positive result. There was a barn effect as barn $B$ had an average Ct value for all treatments and time points of 27 while barn $\mathrm{A}$ had an average 
Ct of $30.2(p<0.05)$. These values correspond to approximately $3 \times 10^{4}$ and $5 \times 10^{5}$ organisms per gram, respectively. There were no statistical differences between treatments but only between sampling days.

Since $\mathrm{ZnAA}$ reduced mucosal lesions, we wondered if ZnAA had an impact on the immune response to infection. We evaluated humoral immune response by testing antibodies against $L$. intracellularis in serum at 14, 21 and 28 dpi. At $14 \mathrm{dpi}$, the ZnAA and ZnAALQ treatments led to a significant increase in the number of animals with antibodies against $L$. intracellularis demonstrating that they seroconverted at an earlier time point than the positive control group $(p<0.05)$ (Figure 3A). This difference was of 94\% seroconversion in the ZnAA group (17 of 18 animals) and $89 \%$ seroconversion (16 of 18 animals) in the ZnAALQ group compared to $50 \%$ seroconversion in the positive control group ( 9 of 18 animals). Statistically significant differences in antibody titers were not observed between the treatment groups when comparing different days to each other (Figure 3B), thus demonstrating that treatment with $\mathrm{ZnAA}$ only reduced the time to seroconversion but did not affect the magnitude of the response. No animals seroconverted in the negative control group.

\section{Quantification of B Cells and T cells}

Since ZnAA had an impact on humoral immunity, next we wanted to evaluate changes to the population of $B$ cells and $\mathrm{T}$ cells at the site of infection. Treatment with either ZnAA or ZnAALQ led to a significant increase in the distribution of $\mathrm{B}$ cells in the lamina propria of the ileum $(p<0.05)$ over time, while pigs in the positive control group showed a trend toward $B$ cell increases in the ileal lamina propria $(p<0.1)$ (Figure 4A).

To quantify $\mathrm{T}$ cells, both crypts infected with $L$. intracellularis and non-infected crypts were evaluated. Among infected crypts, the ZnAA group had significantly more $\mathrm{T}$ cells than the positive control group $(p<0.05)$ at $21 \mathrm{dpi}$ and a trend of higher numbers of $\mathrm{T}$ cells compared to the ZnAALQ group $(p<0.1)$ (Figure $4 \mathrm{~B})$. The ZnAA group was the only one that had a significant increase in $\mathrm{T}$ cells of non-infected crypts compared to the negative group at $21 \mathrm{dpi}(p<0.05)$ (Figure $4 \mathrm{C})$. No other significant differences were found when comparing $\mathrm{T}$ cells of non-infected crypts.

\section{Discussion}

This study was performed to investigate the effect of zinc amino acid complex supplementation on the host immune response to $L$. intracellularis infection and investigate its potential effects in animal production performance during infection. Significant differences were observed using traditional methods of disease measurement including gross and microscopic PPE lesions. ZnAA given in feed or ZnAALQ given in water both led to an overall reduction in the number of animals with gross lesions and a significant decrease in the severity of lesions at $28 \mathrm{dpi}(p<0.05)$ (Figure 1$)$. In terms of
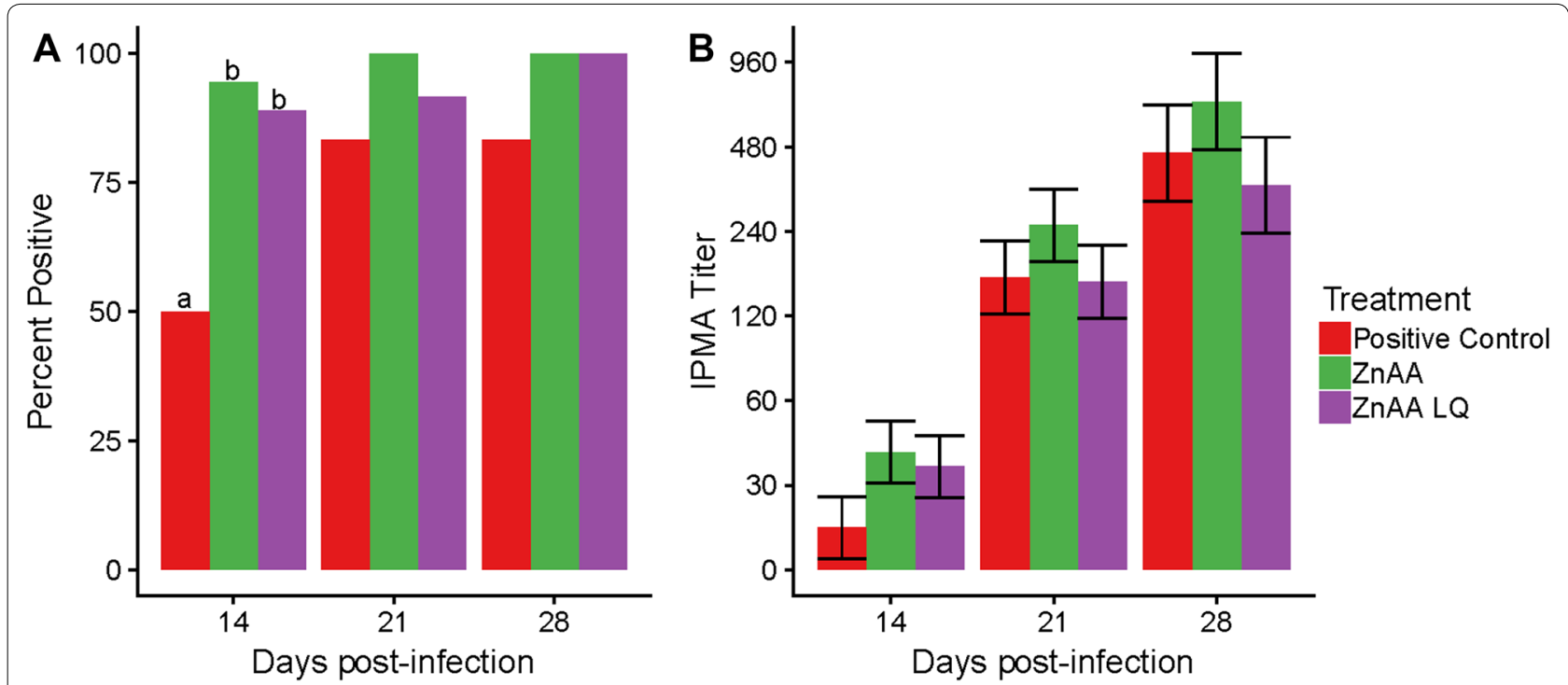

Figure 3 Evaluation of serum antibody responses in animals. At 14, 21 and 28 dpi, the IPMA assay was used to quantify L. intracellularis specific antibodies in serum of animals. A Percent of animals with antibodies in serum against L. intracellularis. B IPMA titer per treatment at different timepoints post-infection. Different letters indicate statistical significance $(p<0.05)$, error bars represent the standard error. No significant differences were found in IPMA titers between treatments but only between time points $(p<0.05)$. 

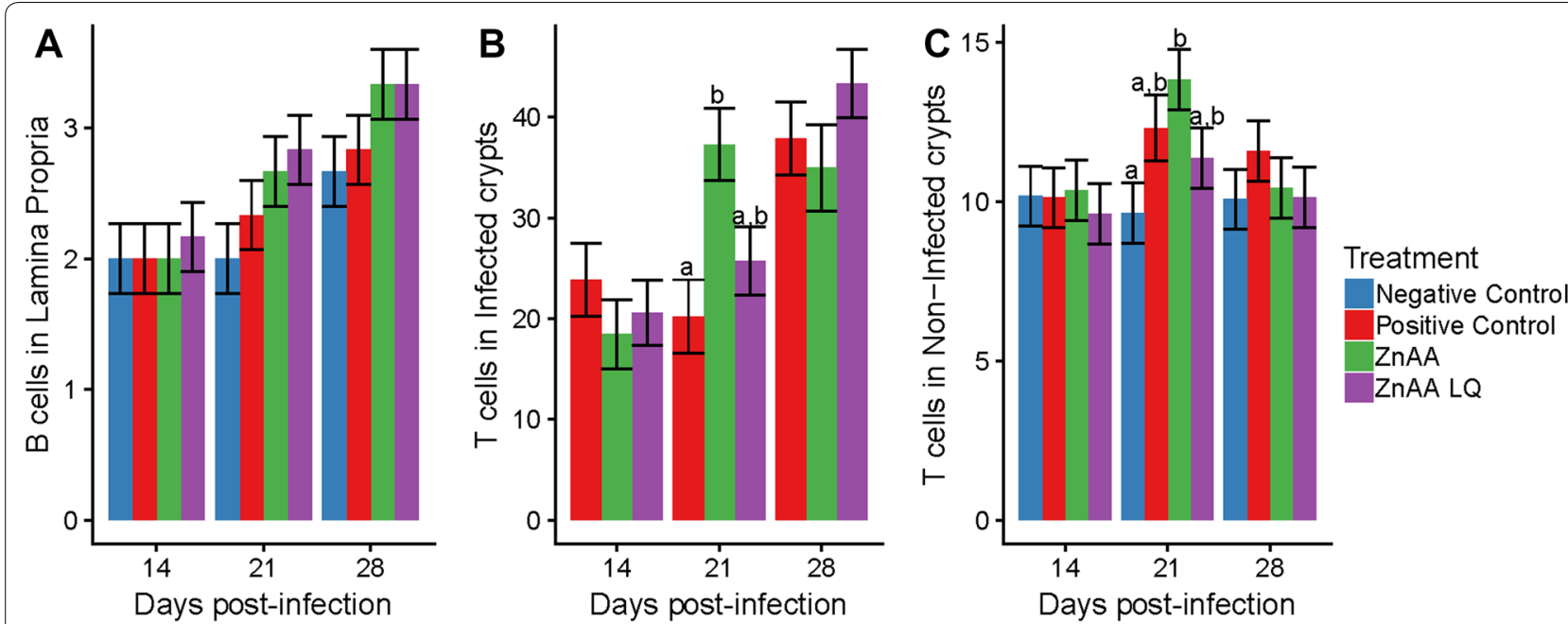

Figure 4 Evaluation of B and T cells in ileum tissue. At 14, 21 and 28 dpi, both B cells and T cells were evaluated in the terminal ileum of all animals. The distribution of B cells was evaluated and given the score of either 1: focal distribution, 2: multifocal distribution, 3: multifocal-to-diffuse distribution, 4: diffuse distribution. T cells were counted in 30 infected and 30 non-infected crypts per animal. A Distribution of B cells in lamina propria. B Number of T cells per infected crypt. C Number of T cells per non-infected crypt. Error bars represent standard error. Different letters indicate statistical significance $(p<0.05)$.

microscopic lesions, $\mathrm{ZnAA}$ given in feed led to a large and significant $(p<0.05)$ decrease (from 83 to $17 \%)$ in the number of animals with lesions at $28 \mathrm{dpi}$ (Figure 2B). This was also the treatment with the least amount of $L$. intracellularis present in ileum tissue at $28 \mathrm{dpi}$, suggesting earlier resolution of lesions (Figure 2A).

In the normal course of experimental infection, the initial detection of $L$. intracellularis inside of enterocytes occurs at approximately $5 \mathrm{dpi}$, and microscopic lesions are detected around $10 \mathrm{dpi}[7,19]$. Disease generally peaks around $21 \mathrm{dpi}$ and at approximately $28 \mathrm{dpi}$ the resolution of lesions generally occurs [7, 19]. The finding that zinc amino acid complex supplementation leads to decreased lesion severity at $28 \mathrm{dpi}$ compared to positive control may indicate that it leads to a shorter disease course and/or a more efficient host response leading to enhanced clearance of infection. This is especially true since animals in the positive control group still had severe lesions at $28 \mathrm{dpi}$. The observed earlier seroconversion post-challenge is consistent with this conclusion. An earlier onset of disease (up to a week) leading to earlier resolution of lesions cannot be ruled out; however.

There were no statistically significant differences between treatments in ADG, ADFI, G:F ratio or water disappearance. Two factors that likely contributed to this were the lack of a clinical impact on overt disease since the challenge dose of L. intracellularis only induced mild disease and the low number of animals used in this trial. The dose given to animals was chosen to induce subclinical disease to better mimic field conditions. Subclinical
L. intracellularis infection does reduce production performance but may require a larger number of animals to reach statistical significance in a study $[8,20]$. Although significant differences in performance were not observed with challenge, L. intracellularis infection was successfully reproduced as confirmed by macroscopic and microscopic lesions, L. intracellularis specific IHC in ileum tissue and antigen specific serum antibody response. Two trends were identified indicating that ZnAA in feed may lead to decreased weight gain and gain to feed ratio. These differences were observed at the time point with the least number of animals per group and should be confirmed with a greater number of animals.

The World Health Organization recommends zinc supplementation for the treatment of diarrhea in children in developing countries. Zinc has been shown to reduce both the duration and severity of diarrhea [21]. This effect includes diarrhea caused by several infectious agents including rotavirus, Vibrio cholerae, Shigella, Salmonella and protozoa such as Cryptosporidium parvum and Giardia lamblia. L. intracellularis infection has not been described in humans. While diets in this study were not deficient in zinc according to the National Research Council (NRC) [14], and contained similar zinc levels in feed, we hypothesized that supplementation with a more bioavailable form of zinc would have a greater effect on the host immune response as compared to zinc sulfate alone. Although clinical disease (i.e. diarrhea) was not observed in this study, ZnAA supplementation still had a positive role in the host response to infection. 
The mechanism(s) responsible for zinc attenuation of enteric disease is not completely understood although zinc is known to have an impact on the immune system $[2,21]$. Similar to the results seen in this study, Rahman et al. found that children infected with Shigella flexneri also had significant increase in seroconversion to $S$. flexneri when given a zinc supplement $(20 \mathrm{mg}$ zinc acetate daily) [22]. A reduction of pathogen load and earlier onset of humoral response was also observed when comparing zinc supplemented to zinc deficient mice infected with Trypanosoma musculi [23]. This may have been one of the factors that led to the observation of beneficial effects of zinc amino acid complex in decreasing lesion severity and decreasing the amount of L. intracellularis present in ileum tissue. Both ZnAA and ZnAALQ led to a significant increase in the number of animals that seroconverted at $14 \mathrm{dpi}$ (Figure 3).

The effects that zinc can have on antibody- and cellmediated immune responses in both humans and animals have been recognized for a long time [24]. Dietary zinc level has been shown to be associated with a host's capacity to produce antigen-specific antibody [25]. Thus it is not surprising that the $\mathrm{ZnAA}$ treatments led to a faster humoral immune response. One of the effects of inadequate zinc levels in the body is lymphopenia [24, 25]. Potentially, this mechanism of increased lymphocytes with zinc supplementation could be related to the observation that both ZnAA treatments led to a significant rise in the distribution of $B$ cells in ileum tissue from 14 to $28 \mathrm{dpi}$. This increase was higher than the one observed in animals that did not receive ZnAA (Figure 4B). Adequate zinc levels have also been shown to have a positive effect on $\mathrm{T}$ cell function and proliferation [26]. ZnAA led to a significant increase in the number of $\mathrm{T}$ cells at $21 \mathrm{dpi}$ in both infected and non-infected tissue $(p<0.05)$ (Figures $4 \mathrm{~B}$ and $\mathrm{C})$. This increase likely also contributed to the superior ability of the animals in this treatment group to resolve lesions and remove $L$. intracellularis from infected tissue.

Both the positive control group and the ZnAA in feed group received the same calculated concentration of zinc in their diet (125 ppm, Table 1) and assayed zinc values were similar. Yet these treatments led to very different responses in pigs when challenged with $L$. intracellularis. Zinc is a trace mineral that is not efficiently stored in the body, thus daily intake is required to achieve adequate steady-state concentrations to support all its functions [2]. It is likely that the zinc amino acid complex led to superior supplementation than zinc alone in the sulfate form, as has been suggested in previous studies $[9,10]$.

Overall, this study demonstrated the impact of zinc amino acid complex supplementation on the host response to $L$. intracellularis infection. Decreases in gross and microscopic lesions were observed at $28 \mathrm{dpi}$, along with increased seroconversion at $14 \mathrm{dpi}$ compared to the group that was challenged and supplemented with inorganic zinc alone. Although benefits were observed only at specific timepoints, these results indicate that zinc supplementation in the form of amino acid complex provided in water or in feed aid the pig immune response to infection and lead to decreased number and severity of PPE lesions. Given the ban of antibiotics as growth promoters in the US and Europe, alternatives to improve animal growth including effective ways to prevent or treat certain infections are needed. The results presented here demonstrate that the possible usage of zinc amino acid complexes may be one alternative.

\section{Additional files}

Additional file 1. Average daily feed intake measured in different treatment groups throughout experiment. The amount of feed (in $\mathrm{Kg}$ ) consumed per animal was calculated by measuring the difference of feed consumed between each week dividing the difference by 7 to obtain daily averages for each week of the trial. Numbers were divided by the number of animals per pen to obtain an average per animal. Error bars represent standard error. No significant differences were found between treatments but only between time points $(p<0.05)$.

Additional file 2. Gain to feed ratio measured in different treatment groups throughout experiment. Gain to feed ratio was obtained by dividing average daily gain by average daily feed intake values. Error bars represent standard error. No significant differences were found between treatments but only between time points $(p<0.05)$.

Additional file 3. Water disappearance measured in different treatment groups throughout experiment. Recording of water provided was performed daily. Water disappearance was calculated by measuring the difference of water consumed between each week dividing the difference by 7 to obtain daily averages for each week of the trial. This value was then divided by the number of animals per pen to obtain a per animal average. Error bars represent standard error. No significant differences were found between treatments but only between time points $(p<0.05)$.

Additional file 4. PCR CT values measured in different treatment groups throughout experiment. Real time PCR was used to estimate the quantity of $L$. intracellularis shed in feces at different time points postinfection. Error bars represent standard error. No significant differences were found between treatments but only between time points $(p<0.05)$.

\section{Abbreviations}

PPE: porcine proliferative enteropathy; PIA: porcine intestinal adenomatosis; PHE: proliferative hemorrhagic enteropathy; ZnAA: zinc amino acid complex formulation provided in feed; ZnAALQ: zinc amino acid complex formulation provided in water; ADG: average daily weight gain; ADFI: average daily feed intake; F:G: feed to gain ratio; HE: hematoxylin and eosin; IHC: immunohistochemistry; IPMA: immunoperoxidase monolayer assay; dpi: days post-infection; NRC: National Research Council.

\section{Competing interests}

ZJR is employed by Zinpro, the company that commercializes the zinc amino acid complex tested.

\section{Authors' contributions}

FLL, CJG, JT, REl, ZJR, NLW, AM were involved in hypothesis generation and experimental design. Animal challenge, clinical and gross lesion evaluation were performed by NLW, AM. Counting of T cells, B cells was performed by EV 
and FV. Microscopic lesion evaluation was performed by: FLL, EV, FAV. Statistical tests were performed by AR. Data interpretation and analysis performed by FLL, CJG, EV, FAV, REI. FLL wrote the manuscript. All authors read and approved the final manuscript.

\section{Acknowledgements}

We wish to thank Jan Shivers and Molly Uaje for staining of histological of tissue. We would also like to thank Robin Winsher for her technical assistance.

\section{Ethics approval and consent to participate}

The animal protocol used was approved by the Swine Services Unlimited Inc. Institutional Animal Care and Use Committee and all experiments were performed in accordance with relevant guidelines and regulations.

\section{Funding}

This project was funded by a grant from Zinpro Corporation. Zinpro Corporation contributed to the design of the study and editing of the manuscript but was not involved in collection, analysis, and interpretation of data.

\section{Author details}

${ }^{1}$ Department of Veterinary and Biomedical Sciences, College of Veterinary Medicine, University of Minnesota, St. Paul, MN, USA. ${ }^{2}$ Department of Veterinary Population Medicine, College of Veterinary Medicine, University of Minnesota, St. Paul, MN, USA. ${ }^{3}$ Swine Services Unlimited Inc., Rice, MN, USA.

${ }^{4}$ Research and Nutritional Services, Zinpro Corporation, Eden Prairie, MN, USA.

\section{Publisher's Note}

Springer Nature remains neutral with regard to jurisdictional claims in published maps and institutional affiliations.

Received: 28 March 2018 Accepted: 1 August 2018

Published online: 10 September 2018

\section{References}

1. Tuerk MJ, Fazel N (2009) Zinc deficiency. Curr Opin Gastroenterol 25:136-143

2. Bonaventura $P$, Benedetti $G$, Albarède F, Miossec $P$ (2015) Zinc and its role in immunity and inflammation. Autoimmun Rev 14:277-285

3. Alam AN, Sarker SA, Wahed MA, Khatun M, Rahaman MM (1994) Enteric protein loss and intestinal permeability changes in children during acute shigellosis and after recovery: effect of zinc supplementation. Gut 35:1707-1712

4. Cario E, Jung S, Harder d'Heureuse J, Schulte C, Sturm A, Wiedenmann B, Goebell H, Dignass AU (2000) Effects of exogenous zinc supplementation on intestinal epithelial repair in vitro. Eur J Clin Invest 30:419-428

5. Pearce SC, Sanz Fernandez MV, Torrison J, Wilson ME, Baumgard LH, Gabler NK (2015) Dietary organic zinc attenuates heat stress-induced changes in pig intestinal integrity and metabolism. J Anim Sci 93:4702-4713

6. Lawson GHK, Gebhart CJ (2000) Proliferative enteropathy. J Comp Pathol 122:77-100

7. Vannucci FA, Gebhart CJ (2014) Recent advances in understanding the pathogenesis of Lawsonia intracellularis infections. Vet Pathol 51:465-477

8. Paradis M, Gebhart CJ, Toole D, Vessie G, Apm D, Winkelman NL, Bauer SA, Wilson JB, Mcclure CA (2012) Subclinical ileitis: diagnostic and performance parameters in a multi-dose mucosal homogenate challenge model. J Swine Heal Prod 20:137-141

9. Sauer AK, Pfaender S, Hagmeyer S, Tarana L, Mattes AK, Briel F, Küry S, Boeckers TM, Grabrucker AM (2017) Characterization of zinc amino acid complexes for zinc delivery in vitro using Caco-2 cells and enterocytes from hiPSC. Biometals 30:643-661

10. Wedekind KJ, Hortin AE, Baker DH (1992) Methodology for assessing zinc bioavailability: efficacy estimates for zinc methionine, zinc sulphate and zinc oxide. J Anim Sci 70:178-187

11. Davin R, Manzanilla EG, Klasing KC, Pérez JF (2013) Effect of weaning and in-feed high doses of zinc oxide on zinc levels in different body compartments of piglets. J Anim Physiol Anim Nutr (Berl) 97:6-12

12. Yazdankhah S, Rudi K, Bernhoft A (2014) Zinc and copper in animal feed-development of resistance and co-resistance to antimicrobial agents in bacteria of animal origin. Microb Ecol Health Dis 25:25862

13. Payne RL, Bidner TD, Fakler TM, Southern LL (2006) Growth and intestinal morphology of pigs from sows fed two zinc sources during gestation and lactation. J Anim Sci 84:2141-2149

14. Council NR (2012) Nutrient requirements of swine, 11 th edn. The National Academies Press, Washington

15. Winkelman NL, Crane JP, Elfring GD, Kratzer DD, Meeuwse DM, Dame KJ, Buckham SL, Gebhart CJ (2002) Lincomycin-medicated feed for the control of porcine proliferative enteropathy (ileitis) in swine. J Swine Heal Prod 10:107-111

16. Macintyre N, Smith DG, Shaw DJ, Thomson JR, Rhind SM (2003) Immunopathogenesis of experimentally induced proliferative enteropathy in pigs. Vet Pathol 40:421-432

17. Guedes RMC, Gebhart CJ, Winkelman NL, Mackie-Nuss RAC, Marsteller TA, Deen J (2002) Comparison of different methods for diagnosis of porcine proliferative enteropathy. Can J Vet Res 66:99-107

18. Guedes RMC, Gebhart CJ, Deen J, Winkelman NL (2002) Validation of an immunoperoxidase monolayer assay as a serologic test for porcine proliferative enteropathy. J Vet Diagn Invest 14:528-530

19. Gebhart CJ, Guedes RMC (2010) Lawsonia intracellularis. In: Gyles CL, Prescott JF, Songer G, Thoen CO (eds) Pathogenesis of bacterial infections in animals, 10th edn. Wiley-Blackwell, Hoboken, pp 503-512

20. Helm ET, Outhouse AC, Schwartz KJ, Dekkers JCM, Steven M, Rauw WM Gabler NK (2018) Impact of Mycoplasma hyopneumoniae and Lawsonia intracellularis on the performance of pigs divergently selected for feed efficiency. J Anim Sci 96:462-472

21. Thawani V, Bajait C (2011) Role of zinc in pediatric diarrhea. Indian J Pharmacol 43:232-235

22. Rahman MJ, Sarker P, Roy SK, Ahmad SM, Chisti J, Azim T, Mathan M, Sack D, Andersson J, Raqib R (2005) Effects of zinc supplementation as adjunct therapy on the systemic immune responses in shigellosis. Am J Clin Nutr 81:495-502

23. Lee CM, Humphrey PA, Aboko-cole GF (1983) Interaction of nutrition and infection: effect of zinc deficiency on immunoglobulin levels in Trypanosoma musculi infection. J Natl Med Assoc 75:677-682

24. Fraker PJ, King LE, Laakko T, Vollmer TL (2000) The dynamic link between the integrity of the immune system and zinc status. J Nutr 130:1399-1406

25. Fraker PJ, King LE (2004) Reprogramming of the immune system during zinc deficiency. Annu Rev Nutr 24:277-298

26. Haase H, Rink L (2014) Multiple impacts of zinc on immune function. Metallomics 6:1175-1180 